\section{Yi Pan, MD \\ Department of Radiation Oncology, Guangdong Lung Cancer Institute, Guangdong Provincial People's Hospital, Guangdong Academy of Medical Sciences, School of Medicine, South China University of Technology, Guangzhou, PR China}

\section{Peixin Tan, MD}

Department of Radiation Oncology, Guangdong Lung Cancer Institute, Guangdong Provincial People's Hospital, Guangdong Academy of Medical

Sciences, School of Medicine, South China University of Technology, Guangzhou, PR China

\section{Songxi Xie, MD}

Department of Radiation Oncology, Guangdong

Provincial People's Hospital, Guangdong Academy

of Medical Sciences, School of Medicine, South China

University of Technology, Guangzhou, PR China

\section{Jiewen Chen, MMed}

Department of Radiation Oncology, Guangdong Provincial People's Hospital, Guangdong Academy of Medical Sciences, School of Medicine, South China University of Technology, Guangzhou, PR China

\section{Yi-Long Wu, MD}

Guangdong Lung Cancer Institute, Guangdong Provincial People's Hospital, Guangdong Academy of Medical Sciences, School of Medicine, South China University of Technology, Guangzhou, PR China

\title{
Pseudopathologic vertebral body enhancement
}

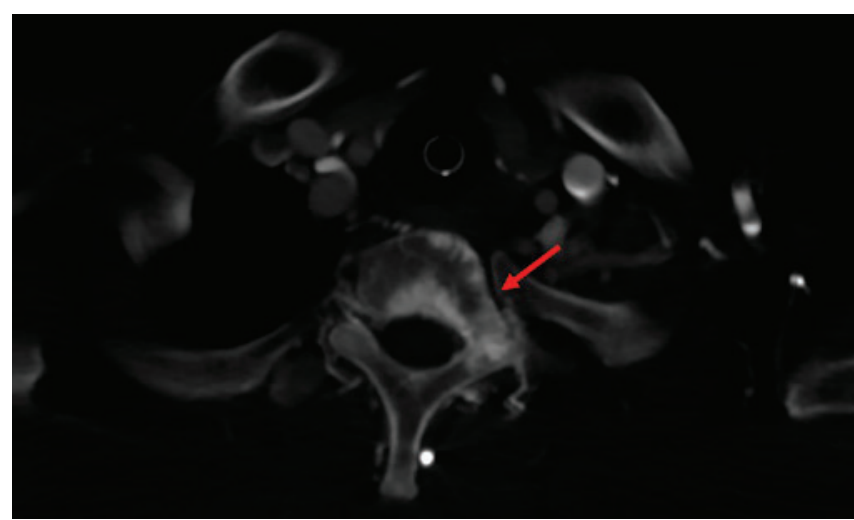

Figure 1. Contrast-enhanced thoracic computed tomography showed sclerotic enhancement of the T1 vertebral body (red arrow).

\begin{abstract}
A 45-YEAR-OLD MAn presented to the emergency department with severe dyspnea and cough that had gradually worsened over the past 3 months. His medical history was notable for stage IIA esophageal squamous cell carcinoma that had been treated with esophagectomy 15 years ago; and 13 years ago, he had developed mediastinal lymph node metastasis and had received chemoradiotherapy. He had been a heavy smoker and drinker but had quit 15 years ago.

Emergency contrast-enhanced thoracic computed tomography (CT) revealed a tumor mass in the lower trachea, sclerotic enhancement of the $\mathrm{T} 1$ vertebral body (Figure 1) and from the C2 to T2 vertebral bodies, and thrombosis in the left brachiocephalic vein (Figure 2). The patient rapidly developed type 2 respiratory failure (defined as a $\mathrm{PaO}_{2}<8.0 \mathrm{kPa}$ and a $\mathrm{PaCO}_{2}$ $>6.0 \mathrm{kPa}$ ) and underwent bedside fiberoptic bronchoscopy, which revealed an obstructive tumor in the lower trachea that had invaded the carina and both
\end{abstract}

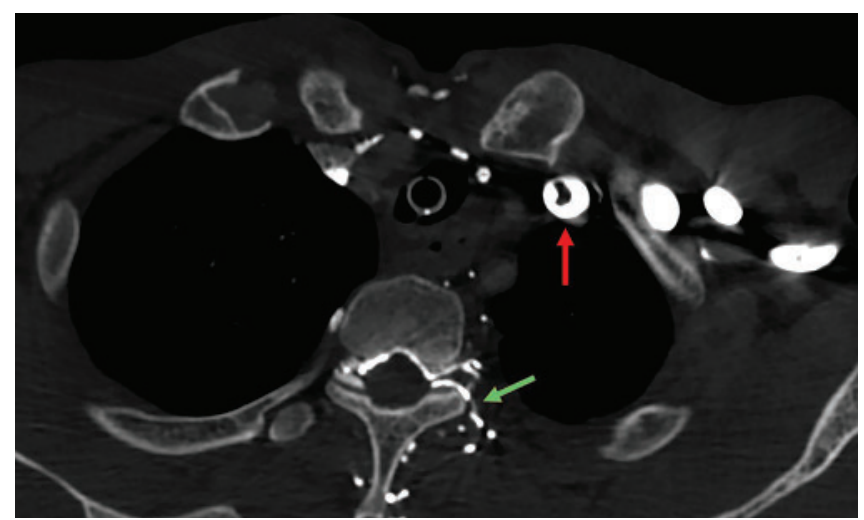

Figure 2. Contrast-enhanced computed tomography noted thrombosis in the left brachiocephalic vein (red arrow) and contrast filling of the paravertebral veins, usually occult on contrast enhancement (green arrow).

the left and right main bronchi. Bronchoscopy-guided radiofrequency ablation was performed to relieve airway obstruction. Moderately differentiated squamous cell carcinoma was confirmed by endobronchial biopsy study.

The patient's dyspnea improved after bronchoscopic therapy. Repeat CT 5 days later showed no evidence of the vertebral body enhancement (Figure 3 ). Esophagoscopy showed an anastomotic stenosis 24 $\mathrm{cm}$ from the incisors and smooth mucosa in the esophagus. Anastomotic stenosis was consistent with the tumor location in the lower trachea and carina. Esophageal stenosis and smooth esophageal mucosa demonstrated external tumor compression of the esophagus, which did not support the diagnosis of esophageal carcinoma.

Based on the patient's smoking and irradiation history and the long interval between the last anticancer treatment and the appearance of the second tumor, the obstructive tumor was diagnosed as a second primary bronchogenic carcinoma.

The patient underwent disease staging with posi- 
tron emission tomography CT, which showed increased 18F-fluorodeoxyglucose uptake at the back wall of the trachea alone, indicating that the tumor was localized.

\section{TREATMENT}

The patient received 4 cycles of capecitabine and anlotinib, followed by 4 cycles of anlotinib. This achieved a partial response. At last follow-up, the patient was alive and without disease progression.

\section{PSEUDOPATHOLOGIC VERTEBRAL BODY ENHANCEMENT}

The differential diagnosis of sclerotic lesions on contrast-enhanced CT includes tumor metastasis, mastocytosis, sarcoidosis, osteomyelitis, lymphoma, Paget disease, and pseudopathologic vertebral body enhancement. For patients with a history of cancer, the leading cause is metastatic malignancy.

A limited number of case reports showed that pseudopathologic vertebral body enhancement may exist in the presence of obstruction of the superior vena cava or brachiocephalic vein. This is uncommon and easily misdiagnosed as sclerotic osseous metastasis in clinical practice. ${ }^{1,2}$

The mechanism of pseudopathologic sclerotic enhancement of vertebral bodies is not well characterized. However, an elevated venous pressure that induced contrast agent reflux into the intravertebral venous plexus was proposed as a main reason. ${ }^{3}$

In our patient, narrowing of the left brachiocephalic vein proximal to the superior vena cava resulted in elevated venous pressure and blood flow into the vertebral venous plexus, causing reflux of contrast agent. ${ }^{1,4}$ Sclerotic enhancement of the vertebral bodies and vertebral venous collaterals, which are usually occult

\section{REFERENCES}

1. Berritto D, Abboud S, Kosmas C, Riherd D, Robbin M. Vertebral body enhancement mimicking sclerotic osseous lesions in the setting of bilateral brachiocephalic vein thrombosis. Skeletal Radiol 2015; 44(2):303-305. doi:10.1007/s00256-014-2037-9

2. Thomas N, Oliver TB, Sudarshan T. Vanishing bone metastases-a pitfall in the interpretation of contrast enhanced CT in patients with superior vena cava obstruction. Br J Radiol 2011; 84(1005):e176e178. doi:10.1259/bjr/50676625

3. Kim YK, Sung YM, Hwang KH, Cho EK, Choi HY. Pseudopathologic vertebral body enhancement in the presence of superior vena cava

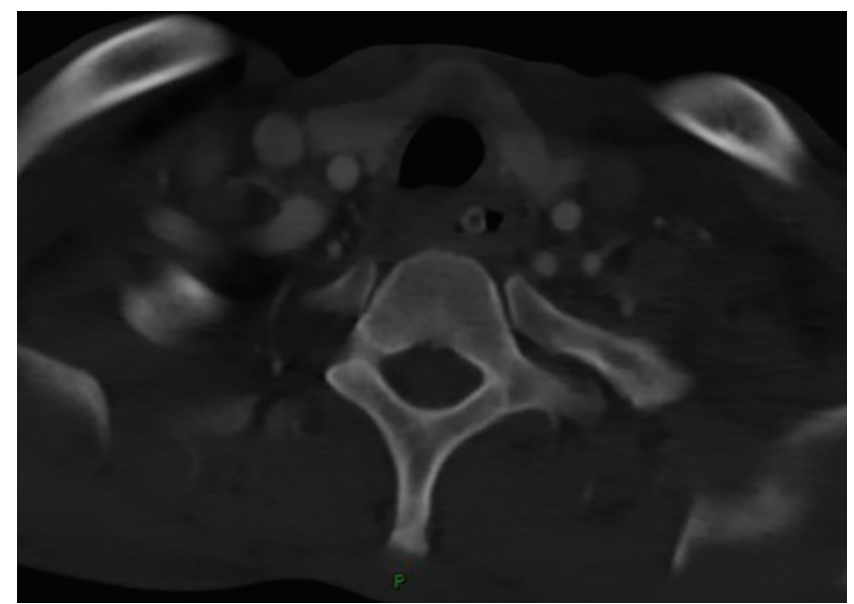

Figure 3. Repeat computed tomography showed the disappearance of the sclerotic vertebral body enhancement.

on CT, were seen. Eight consecutive vertebral bodies were involved, which is uncommon with metastasis. However, when the contrast agent was injected into the contralateral arm during the second CT, the vertebra enhancement disappeared (Figure 3).

Pseudopathologic vertebral body enhancement due to brachiocephalic vein narrowing is rare. However, sclerotic bone metastasis based on contrast-enhanced CT should prompt a careful evaluation when narrowing or obstruction of the brachiocephalic vein and paravertebral collateral veins is present on imaging. Spine magnetic resonance imaging or positron emission tomography CT should be performed to confirm the diagnosis.

\section{DISCLOSURES}

The authors report no relevant financial relationships which, in the context of their contributions, could be perceived as a potential conflict of interest.

obstruction on computed tomography. Spine J 2015; 15(6):12951301. doi:10.1016/j.spinee.2013.07.440

4. Chen JY, Mamourian AC, Messe SR, Wolf RL. Pseudopathologic brain parenchymal enhancement due to venous reflux from leftsided injection and brachiocephalic vein narrowing. AJNR Am J Neuroradiol 2010; 31(1):86-87. doi:10.3174/ajnr.A1688

Address: Yi Pan, MD, Department of Radiation Oncology, Guangdong Lung Cancer Institute, Guangdong Provincial People's Hospital, Guangdong Academy of Medical Sciences, School of Medicine, South China University of Technology, 106 Zhongshan Er Rd, Guangzhou, 510080, PR China; panyiff01@163.com 